\title{
The interaction between brain-derived neurotrophic factor (BDNF) Val66met polymorphism and dietary indices, The Healthy Eating Index (HEI), The Diet Quality Index-International (DQI-I), and Phytochemical index (PI), on anthropometric indices, inflammatory, and oxidative stress markers in patient with type2 diabetes
}

Fariba Koohdani ( $\nabla$ fkoohdan@tums.ac.ir)

Tehran University of Medical Sciences https://orcid.org/0000-0002-7108-5350

Zeinab Naeini

Tehran University of Medical Sciences

Negin Aghazadeh

Azad University: Islamic Azad University

Elmira Karimi

Tehran University of Medical Sciences

Zahra Esmaeily

Tehran University of Medical Sciences

Faezeh Abaj

Tehran University of Medical Sciences

Masoumeh Rafiee

Isfahan University of Medical Sciences

Research

Keywords: brain-derived neurotrophic factor, Val66met polymorphism, Dietary indices, Biochemical parameters, anthropometric measurements

Posted Date: February 23rd, 2021

DOl: https://doi.org/10.21203/rs.3.rs-239163/v1

License: (c) (i) This work is licensed under a Creative Commons Attribution 4.0 International License.

Read Full License 


\section{Abstract}

Background: Although many studies suggest an association between BDNF Val66Met with eating disorders and obesity especially in type 2 diabetes mellitus (T2DM), the interaction between BDNF Val66Met polymorphism and diet quality indices in diabetic patients have not been investigated yet.

The current study was aimed to evaluate the interaction between the diet quality indices including Healthy Eating Index (HEI), Diet Quality Index-International (DQI-I), Phytochemical Index (PI) and BDNF Val66Mat (rs6265) polymorphism on markers of inflammation, and oxidative stress in T2DM patients.

Methods: This current cross-sectional study was conducted on 634 Iranian T2DM patients aged 35-65 years of both genders were randomly recruited. Dietary intakes was estimated by a food frequency questionnaire (FFQ) with 148 food items. All participants were categorized into three categories, based on DQI, HEl, and PI scores. The interactions were tested using analysis of covariance (ANCOVA) in adjusted and unadjusted models.

Results: There were significant gene-diet interactions between BDNF Val66Met polymorphism and HEl, DQI, and PI scores in modulating body mass index (BMI) and waist circumference (WC) values. Our results showed that higher scores for all diet-quality indices were significantly associated with a lower $B M I$ and $W C$ values after adjustment ( $P$ Interactions $<0.05)$. We also observed a significant interaction between the DQI scores and Val66Met polymorphism on level of superoxide dismutase (SOD) ( $\mathrm{p}$-interaction= 0.01). The highest quartile of DQI was associated with elevated level of SOD in the Val/Met and Met/Met genotype group. Moreover, the interaction between the DQI scores and Val66Met polymorphism on total antioxidant capacity (TAC) tended to be significant.

Conclusions: Higher scores for all diet-quality indices were significantly associated with a lower BMI and WC values. There were significant differences for PTX and PGF2A among HEl tertiles. The interaction between the polymorphism and DQI on TAC and SOD levels were significant.

\section{Introduction}

Type 2 diabetes mellitus is rising rapidly especially in Asia and it is estimated that the prevalence of this disease in Iran will increase to 9.2 million by 2030 (1). It may be related to wrong lifestyles and dietary patterns such as consuming a high-fat diet and lack of exercise and can lead to a wide variety of complications (2). There is an important relationship between hyperglycemia, hyperglycemic-induced oxidative stress, inflammation, and the progression of T2DM. Oxidative stress activates the production of inflammatory mediators and inflammation triggered by oxidative stress stimulates the generation of reactive oxygen species (ROSs) (3). Genetic and environmental factors contribute significantly to the development of type 2 diabetes. Diet is the greatest factor in lifestyle that can greatly affect the incidence and the progression of T2DM and its long-term problems (4). Epidemiologic studies have shown a positive relation between dietary factors and chronic diseases. To determine the nutritional status of individual, dietary indices have been developed $(5,6) \mathrm{HEl}$, a measure for evaluating alignment of dietary 
intake and DQI, focuses on adequacy, variety, balance, and moderation are two important indices which encourage people to eat a diversified, balanced, and healthy diet including vegetables, fruits and healthier types of fat. The HEl is developed by the United States Department of Agriculture (USDA) and based on the food guide pyramid. A study in Iranian women showed that a higher adherence to the HEI was inversely linked with the risk of metabolic syndrome and another study in elderly Iranian people revealed that adherence to the HEI was related to a lower risk of cardiovascular disease (CVD) risk factors. To the best of our knowledge, few studies have evaluated the association of the DQI and HEI with CVD risk factors among diabetic patients. Controversly, a cross-sectional study on T2DM women showed no association between HEI, DQI-I, and CVD risk factors (7). Genome-wide association studies (GWAS) have uncovered genes associated with T2DM in different populations, including European and Asian populations (2). One of them is brain-derived neurotrophic factor (BDNF) that has long been known for its important role in neuronal differentiation and growth. Recent studies suggest that BDNF is involved in the regulation of eating behavior and energy expenditure in animals and humans. Elevated level of circulating BDNF is thought to contribute to inhibit food intake and increases physical activity (8).

The human BDNF gene, extending over $70 \mathrm{~kb}$, is located on chromosome 11, region p13-14. There are hundreds of common single-nucleotide polymorphisms (SNPs) in the BDNF gene (9). Val66Met polymorphism (rs6265) is a common and functional SNP which results in a valine (Val) to methionine (Met) substitution at codon $66(8,9)$. Many studies show that BDNF can be related to eating behavior, food intake and modulation of the secretion of insulin, leptin, ghrelin, and pro inflammatory cytokines. These results suggest that BDNF may be a factor which develop obesity and T2DM in humans $(8,10,11)$. Most of studies suggest an association between BDNF Val66Met with eating disorders and obesity (12). The present study aimed to investigate the association of diet quality indices including the DQI, HEl, and PI with some of risk factors such as oxidative stress and inflammatory markers among T2DM individuals.

\section{Methods}

\section{Sample size calculation}

In this paper, the Sample size was calculated according to following formula:

$N=\left(\left(\left[(Z 1-a+Z 1-\beta) \times \sqrt{ } 1-r^{2}\right] / r\right)^{2}+2\right)$, When $r=0.15 \beta=0.95$ and $a=0.05$, the $N$ was equal to 634 .

\section{Study design and subjects}

For this cross-sectional study, 636 T2DM patients aged 35-65 years of both genders (252 men and 384 women), were randomly recruited from diabetes referral centers including Gabric Diabetes Association, Iranian Diabetes Society, and other health centers in Tehran during June 2011 to October 2012. All the subjects had either fasting blood glucose levels of $\geq 126 \mathrm{mg} / \mathrm{dl}$ or were under treatment with medication (oral) by a physician. Persons under 35 or over 65 years of age, insulin administrating patients, pregnant or lactating women, were excluded. The current study was approved by the ethics committee of Tehran 
University of Medical Sciences (TUMS) (Ethnic number: IR.TUMS.VCR.REC.1395.15060) and the informed consent form was obtained from all participants. Demographic and general data including age, gender, job, and education status, smoking, and alcohol abuse, lipid-lowering drug consumption, family history of T2DM, and other diseases were collected through interviews.

\section{Assessment of anthropometric measurement and physical activity}

Anthropometric data were measured by standard methods. Weight was measured in the fasting state, with the minimal clothing, by using Seca falcon scales, with an accuracy of $100 \mathrm{~g}$. Height was measured using a Seca height gauge with an accuracy of $0.5 \mathrm{~cm}$ according to standard protocols (13). BMI was calculated as body weight $(\mathrm{kg})$ divided by the square of the height $(\mathrm{m} 2)$. Physical activity was calculated as metabolic equivalent of task (MET h/day) (14) by a validated and reliable physical activity questionnaire $(15,16)$.

\section{Assessment of dietary intake}

The participant's usual dietary intake during the last year was evaluated through face-to-face interviews conducted by a trained dietitian using a semi quantitative food frequency questionnaire (FFQ) for 148 food items. This questionnaire was validated by Esmaillzadeh et al (17). The subjects were asked to report the frequency of food item consumption in a day, a week, a month or a year. The amounts listed for each food were converted to grams per day using household measures (18). Nutritionist III software (version 7.0, N-Squared Computing) was employed to assess the energy and nutrient intake.

\section{Assessment of dietary indices}

Three dietary indices were used for evaluating diet quality: The Healthy Eating Index (HEI), a measure for evaluating alignment of dietary intake according to the 2015-2020 dietary guidelines for Americans (DGA) based on a $1000 \mathrm{kcal} /$ day diet. HEl-2015 score ranged from 0 to 100 and consisted of 13 components (total fruits, whole fruits, total vegetables, greens and beans, seafood or plant protein, and total protein foods can receive a score ranging from 0 to 5 , whole grains, dairy, fatty acids can receive a score ranging from 0 to 5 and refined grains, saturated fats, sodium and added sugars are moderated (higher intakes receive lower scores) (19). We calculated the score based on responses from the FFQs.

The Diet Quality Index-International (DQI-I) was a second dietary measurement which focuses on four main aspects of a healthy diet (variety, adequacy, moderation, and overall balance). The score for each category is calculated as the sum of the scores for each component in that category. Overall food group variety including meat/poultry/fish/eggs/dairy/beans/grain/fruit/vegetable (0-15 points) and Withingroup variety for protein source including meat/poultry/fish/dairy/beans/eggs (0-5 points) , protein sources, vegetable, fruit, grain, fiber, protein, iron, calcium, vitamin $\mathrm{C}$ group (0-5 points), moderation foods such as total fat, saturated fat, cholesterol, sodium and junk foods ( $0-30$ points), macronutrient and fatty acid (0-10 points) (20). The total DQI-I score (ranging from 0 to 100 points) is the sum of the scores for the four categories. 
Moreover, Phytochemical Index (PI), defined as the percentage of dietary calories derived from foods rich in phytochemicals including fruits, vegetables, whole grains, nuts, seeds, vegetable juices, soy products, olive oil. The dietary phytochemical index (DPI) was calculated based on the modified method previously developed by McCarty (21); [PI= (phytochemical-rich foods $\mathrm{g} / \mathrm{d} /$ total food intake $\mathrm{g} / \mathrm{d}$ ) $\times 100]$.

\section{Biochemical assessment and genotyping}

Venous blood samples were collected after 12-h overnight fasting. The total antioxidant capacity (TAC) of serum was measured by spectrophotometry. TAC measurement evaluates the overall power of all antioxidants in the body $(21,22)$ Serum enzymatic activity of superoxide dismutase (SOD), as an enzymatic antioxidant (22) was estimated by colorimetric method (Cayman Chemical Company, USA). Interleukin-18 (IL-18), Pentrexin-3 (PTX3), and 8-isoprostane F2a (PGF2a) were measured using ELISA method (Shanghai Crystal Day Biotech Co., Ltd). The sensitivity of IL-18 and PTX3 ELISA kit was $28 \mathrm{ng} / \mathrm{I}$ and $0.05 \mathrm{ng} / \mathrm{ml}$, respectively. Genomic DNA was isolated from whole blood using salting-out extraction method (23). Polymerase chain reaction (PCR) was used for genotyping the BDNF Val66met, followed by $8 \%$ polyacrylamide gel electrophoresis.

\section{Statistical analysis}

Normal distribution of data was measured using Kolmogorov-Simonov test. Logarithmic transformations were applied to variables with skewed distribution. The subjects were divided in to two genotype groups: Val/Val and Val/Met, Met/Met. The data were presented as frequency (\%) for categorical variables and as mean \pm SD for continuous variables.

Independent T-test was used to compare the quantitative variables between the two groups and chisquare test was used In order to compare the qualitative variables.

The association between diet quality indices and anthropometric or biochemical parameters were evaluated by one-way analysis of variance (ANOVA).

The interaction between BDNF Val/Met polymorphism and DEI, DQI, and PI on anthropometric indices, serum inflammatory, and oxidative stress markers were tested using analysis of covariance (ANCOVA) test in two multivariate interaction models, before and after adjustment for potential confounders including age, sexuality, smoking, alcohol consumption, and physical activity. The data were analyzed by IBM SPSS (SPSS Inc., Chicago, IL, USA, version 26) and $P$ value $<0.05$ was considered as statistically significant.

\section{Result}

This cross-sectional study was conducted on 634 T2DM patients with sex distribution of $39.7 \%$ and $60.3 \%$ in men and women respectively. Val66Met genotype prevalence among study participants was as follow: The Met allele carrier group had a frequency of $44.2 \%$ (Val/Met+ Met/Met) and accordingly the major allele frequency was 55.8\% (Val/Val). Anthropometric, general characteristics, and nutrient 
intakes of participants across BDNF Val66Met genotypes are presented in Table1. Comparison of clinical parameters of participants according to BDNF Val66Met genotypes are presented in Table2. There was no significant difference regarding these parameters across BDNF Val66Met genotypes among the groups. BDNF Met allele carriers (Met/Met+ Val/Met) had nominally significantly higher energy intakes and correspondingly higher intakes of all nutrients than those with BDNF Val/Val.

All participants were categorized into tertiles, based on DQI, HEI, and DPI scores. There were statistically significant differences for $\mathrm{BMI}$ among $D Q I$ tertiles $(p=0.02)$, HEI tertiles $(\mathrm{p}=0.01)$, and DPI tertiles $(p=0.03)$. Also, there was a marginal significant difference between HEl tertiles for WC $(p=0.01)$ and it is tended to be significant in DPI tertiles too ( $p=0.08)$. Significant difference were found between $\mathrm{HEI}$ tertile groups for PTX3 $(p=0.02)$ and PGF2a $(p=0.03)$.

Table 3 shows analysis for general and biochemical markers in different tertiles of dietary quality indices. The results of the interaction analysis between Val66Met polymorphism and dietary indices on anthropometric data, inflammatory, and oxidative stress parameters revealed Significant interactions between HEl score and Val66Met polymorphism for BMI and WC ( $p=0.001$ and $p=0.0$, respectively). Our results showed that higher scores for all diet-quality indices were significantly associated with a lowerbody mass index and waist circumference values and this association remained consistently significant even after adjusting for various potential confounders including age, weight, height, and physical activity.

In addition, significant interactions were observed between SNP Val66Met and DQI, DPI on BMI and WC $(P<0.05)$ after adjustment for the confounding variables. Also the interaction between the polymorphism and DQI on SOD level were statistically significant (p-interaction=0.01). The highest quartile of DQI was associated with elevated level of SOD in the Val/Met and Met/Met genotype group. Moreover, the interaction between the DQI scores and BDNF Val66Met polymorphism on total antioxidant capacity tended to be significant (p-interaction=0.08). Significant interactions between BDNF Val66Met genotypes and tertiles of DQI, HEI, and DPI are shown in Fig. 1, 2, and 3 respectively.

\section{Discussion}

The current study investigated interactions between the DQI, HEI, DPI, and BDNF Val66Met polymorphism on markers of anthropometric parameters, inflammation, and oxidative stress markers in T2DM patients. We found no significant difference for clinical characteristics between different genotypes of Val66Met. Although, our result showed that BDNF Val/Met and Met/Met patients had significantly higher cholesterol intake than those with BDNF Val/Val and there was a significant difference between the genotypes of Val66Met for cholesterol intake $(p=0.0)$.

BDNF variants affected daily dietary intake such as total energy, carbohydrates, fiber, protein, fat in diabetic patients. The Met allele carrier group (Met/Met and Val/Met genotypes) had significantly higher nutritional intakes compared to subjects with Val/Val genotype. This result ties well with a study showed that none of the daily nutrient intake such as energy, fat, carbohydrates, protein... was not significantly 
different by BDNF variants in nondiabetic individuals. However, daily nutrient intakes affected by BDNF Val66Met polymorphism in diabetic patients. Our result showed that BDNF Val/Met and Met/Met patients had significantly higher energy and nutrients intake than those with BDNF Val/Val.

Both human and animal studies suggest that BDNF SNPs play a significant role in the regulation of appetite and nutrient intake $(2,24-26)$. Several studies showed that T2DM patients with Val/Met and Met/Met had higher energy intakes because BDNF depletion is known to result in hyperphagia $(2,27,28)$. They noted significantly lower plasma BDNF levels in diabetic patients and also in the Met allele career group $(11,26,29)$. Some of them reported asignificant relationship between BDNF and obesity or type 2 diabetes development in humans $(10,25,30,31)$.

Our results showed that higher scores for all diet-quality indices were significantly associated with a lowerbody mass index and waist circumference values after adjustment for the confounders. This result was consistent with the findings of a previous meta-analysis that reported the diet quality indices based on dietary guidelines were inversely associated with BMI and WC in most studies (32).

There are only few studies on the gene-nutrient interaction between the BDNF variants and type 2 diabetes $(2,27,28)$.Inflammation is known as a major risk factor of developing type 2 diabetes. Many studies demonstrated increased levels of all markers of inflammation such as: IL-1 $\beta$, IL-6, and C-reactive protein (CRP) are predictive of type 2 diabetes mellitus (33). We evaluated the interaction between dietary indices and BDNF Val66Met polymorphism on inflammatory and oxidative stress markers. No significant interactions were seen between dietary indices and the genotypes of Val66Met on biomarkers of inflammation after adjustment for the variables. However, higher scores for DQI and HEI were associated with a lower levels of interleukin-18 in the both genotypes group and also the highest-quartile HEI score was associated with lower PTX Levels. This result agrees with some previous studies that reported the DQI and HEI scores had little association with inflammation biomarkers (33). Findings indicated that the Val/Val genotype was associated with higher mean CRP levels than those carrying the Met genotype. However, the difference is not significant among the groups. The elevated CRP level predicts adverse cardiovascular events independently. A number of epidemiological studies, also have reported the higher plasma CRP level in the Val/Val genotype. They hypothesized that the Val/Val genotype would be associated with increased severity of coronary artery disease (CAD) and incidence of CVD (34).

A few studies investigated the associations between the $\mathrm{HEl}$ and cardio metabolic risk factors among Iranians reported no significant association between the HEI/AHEI with CRP level and other CVD risk factors (35-37). These findings are similar to previous data showing that these prudent dietary patterns are inversely associated with $\mathrm{BMI}$ and $\mathrm{WC}$, but not with plasma oxidative stress status or inflammation markers $(38,39)$. However, an important finding of this study would be that subjects in the highest quartiles, Q3, of the DPI scores had lower CRP concentrations in the Met-allele carrier group.

No study has been conducted to assess the interaction between diet quality indices and BDNF Val66Met polymorphism in diabetic patients. 
Different than expected, there were no relation between dietary indices and oxidative stress markers. Similar to another study indicates no relationship between the score of HEl and the OS parameters. Furthermore, there was no correlation between the OS parameters and DPI scores (40). One possible cause for the lack of relationship may be the difficulty of in vivo OS measurement, considering the complex network of antioxidant and oxidant and also very short half-lives of free radicals $(40,41)$. Some of studies suggest that dietary intake can modulate antioxidant status after acute consumption of foods rich in antioxidant (42-46).

Furthermore, our results show no significant interactions between DPI and BDNF Val66Met polymorphism on oxidative stress markers. A previous study showed that the PI score was inversely related to oxidative stress, but not with plasma total antioxidant status. An explanation could be that the sources of phytochemicals may vary even among persons with the same PI score (38). Low phytochemical intake is likely related to elevated oxidative stress and inflammation, although these relationships have not yet been clarified (39). Oxidative stress plays a key role in the development of diabetic complications (3).

The elevated level of superoxide dismutase indicates oxidative stress reduction and a decreased level of SOD were found in diabetic tissue and blood in different studies (47). We also

observed a significant interaction between the DQI scores and BDNF Val66Met polymorphism on level of SOD. The highest quartile of DQI was associated with elevated level of SOD in the Val/Met and Met/Met genotype group. Moreover, the interaction between the DQI scores and BDNF Val66Met polymorphism on total antioxidant capacity (TAC) tended to be significant.

We present novel findings regarding the interaction of dietary indices and BDNF Val66Met polymorphism on inflammatory and oxidative stress markers in diabetic individuals. It is also suggested that in future studies, the interaction of this polymorphism and dietary indices on inflammatory and oxidative stress markers to be studied on healthy individuals as well.

The main limitation of this research is that we could not measure blood sugarlevel of participants due to time and budget limitations. So, we missed potentially relevant interactions. Besides, we controlled for several potential confounders, the effects of remaining confounders cannot be ignored. Although, further investigations in different populations are needed to confirm the results of the present study.

\section{Conclusion}

The current study aimed to provide a direct investigation of the interaction between BDNF Val66Met polymorphism and diet quality indices on inflammation and oxidative stress in T2DM patients. Higher scores for all diet-quality indices were significantly associated with a lower BMI and WC values. Also, significant interactions were found between HEI, DQI, DPI scores, and the genotypes of BDNF Val66Met for BMI and WC. There were significant differences for PTX and PGF2 $a$ among HEI tertiles. The interaction between the polymorphism and DQI on TAC and SOD levels were significant. 


\section{Abbreviations}

BDNF Brain-derived neurotrophic factor

BMI Body mass index

CRP C-reactive protein

CVD Cardiovascular disease

DQI Diet quality index-international

HEI Healthy eating index

IL-18 Interleukin-18

PI Phytochemical index

PGF2a, 8-isoprostane

PTX3 Pentrexin-3

SOD Superoxide dismutase

TAC Total antioxidant capacity

T2DM Type 2 diabetes mellitus

WC Waist circumference

\section{Declarations}

\section{Ethics approval and consent to participate}

The current study was approved by the ethics committee of Tehran University of Medical Sciences (TUMS) (Ethnic number: IR.TUMS.VCR.REC.1395.15060)

\section{Consent for publication}

the informed consent form was obtained from all participants.

\section{Availability of data and materials}

The datasets used and/or analysed during the current study are available from the corresponding author on reasonable request. 
The authors report no conflicts of interest.

\section{Funding}

The present study was supported by the Tehran University of Medical Sciences grant no. 97-01-16138100.

\section{Authors' contributions}

The authors' responsibilities were as follows-M.R and F.K: designed research and M.R, F.K, and Z.N: conducted the research. M.R, Z.E, and F.A: collected data; N.A, E.K, M.R, and F.K: participated in data analysis and interpretation; Z.N and M.R: wrote the paper. F.K, M.R, and ZN: had primary responsibility for the final content; Z.N and M.R proofread the manuscript for native English writing; and all authors: read and approved the final manuscript.

\section{Acknowledgments}

The present study was supported by the Tehran University of Medical Sciences grant no. 97-01-16138100.

\section{References}

1. Naeini Z, Toupchian O, Vatannejad A, Sotoudeh G, Teimouri M, Ghorbani M, et al. Effects of DHAenriched fish oil on gene expression levels of p53 and NF-KB and PPAR-y activity in PBMCs of patients with T2DM: A randomized, double-blind, clinical trial. Nutrition, Metabolism and Cardiovascular Diseases. 2020;30(3):441-7.

2. Daily JW, Park S. Interaction of BDNF rs6265 variants and energy and protein intake in the risk for glucose intolerance and type 2 diabetes in middle-aged adults. Nutrition. 2017;33:187-94.

3. Oguntibeju 00. Type 2 diabetes mellitus, oxidative stress and inflammation: examining the links. International journal of physiology, pathophysiology and pharmacology. 2019;11(3):45.

4. Dal S, Sigrist S. The protective effect of antioxidants consumption on diabetes and vascular complications. Diseases. 2016;4(3):24.

5. Chen G-C, Koh W-P, Neelakantan N, Yuan J-M, Qin L-Q, van Dam RM. Diet quality indices and risk of type 2 diabetes mellitus: the Singapore Chinese health study. American journal of epidemiology. 2018;187(12):2651-61.

6. Ozer E. Evaluating dietary quality in diabetes by the Healthy Eating Index. Asia Pacific journal of clinical nutrition. 2013;22(4):620.

7. Daneshzad E, Larijani B, Azadbakht L. Diet quality indices and cardiovascular diseases risk factors among diabetic women. Journal of the Science of Food and Agriculture. 2019;99(13):5926-33.

8. Gunstad J, Schofield P, Paul RH, Spitznagel MB, Cohen RA, Williams LM, et al. BDNF Val66Met polymorphism is associated with body mass index in healthy adults. Neuropsychobiology. 
2006;53(3):153-6.

9. Tudor L, Konjevod M, Nikolac Perkovic M, Svob Strac D, Nedic Erjavec G, Uzun S, et al. Genetic variants of the brain-derived neurotrophic factor and metabolic indices in veterans with posttraumatic stress disorder. Frontiers in psychiatry. 2018;9:637.

10. Zhou J-X, Li H-C, Bai X-J, Chang B-C, Li C-J, Sun P, et al. Functional Val66Met polymorphism of Brainderived neurotrophic factor in type 2 diabetes with depression in Han Chinese subjects. Behavioral and Brain Functions. 2013;9(1):34.

11. Krabbe K, Nielsen A, Krogh-Madsen R, Plomgaard P, Rasmussen P, Erikstrup C, et al. Brain-derived neurotrophic factor (BDNF) and type 2 diabetes. Diabetologia. 2007;50(2):431-8.

12. Ma X-Y, Qiu WQ, Smith CE, Parnell LD, Jiang Z-Y, Ordovas JM, et al. Association between BDNF rs6265 and obesity in the Boston puerto rican health study. Journal of obesity. 2012;2012.

13. Consultation W. Waist circumference and waist-hip ratio. Report of a WHO Expert Consultation Geneva: World Health Organization. 2008;2008:8-11.

14. Ainsworth BE, Haskell WL, Leon AS, Jacobs Jr DR, Montoye HJ, Sallis JF, et al. Compendium of physical activities: classification of energy costs of human physical activities. Medicine and science in sports and exercise. 1993;25(1):71-80.

15. Aadahl M, Jørgensen T. Validation of a new self-report instrument for measuring physical activity. Medicine and science in sports and exercise. 2003;35(7):1196-202.

16. Klishadi R, Khosravi A, Famouri F, Sadeghi M, Shirani S. Assessment of physical activity of adolescents in Isfahan. Journal of Shahrekord University of Medical Sciences. 2001;3(2).

17. Esmaillzadeh A, Mirmiran P, Azizi F. Whole-grain consumption and the metabolic syndrome: a favorable association in Tehranian adults. European journal of clinical nutrition. 2005;59(3):353-62.

18. Rafiee M, Sotoudeh G, Djalali M, Alvandi E, Eshraghian M, Javadi F, et al. The interaction between apolipoprotein $b$ insertion/deletion polymorphism and macronutrient intake on lipid profile and serum leptin and ghrelin levels in type 2 diabetes mellitus patients. European journal of nutrition. 2019;58(3):1055-65.

19. Krebs-Smith SM, Pannucci TE, Subar AF, Kirkpatrick SI, Lerman JL, Tooze JA, et al. Update of the healthy eating index: HEl-2015. Journal of the Academy of Nutrition and Dietetics. 2018;118(9):1591602.

20. Kim S, Haines PS, Siega-Riz AM, Popkin BM. The Diet Quality Index-International (DQI-I) provides an effective tool for cross-national comparison of diet quality as illustrated by China and the United States. The Journal of nutrition. 2003;133(11):3476-84.

21. McCarty MF. Proposal for a dietary “phytochemical index”. Medical hypotheses. 2004;63(5):813-7.

22. Zamani E, Sadrzadeh-Yeganeh H, Sotoudeh G, Keramat L, Eshraghian M, Rafiee M, et al. The interaction between ApoA2- 265T> C polymorphism and dietary fatty acids intake on oxidative stress in patients with type 2 diabetes mellitus. European journal of nutrition. 2017;56(5):1931-8. 
23. Miller S, Dykes D, Polesky H. A simple salting out procedure for extracting DNA from human nucleated cells. Nucleic acids research. 1988;16(3):1215.

24. leraci A, Barbieri SS, Macchi C, Amadio P, Sandrini L, Magni P, et al. BDNF Val66Met polymorphism alters food intake and hypothalamic BDNF expression in mice. Journal of Cellular Physiology. 2020.

25. Singh RB, Takahashi T, Tokunaga M, Wilczynska A, Kim CJ, Meester FD, et al. Effect of brain derived neurotrophic factor, in relation to diet and lifestyle factors, for prevention of neuropsychiatric and vascular diseases and diabetes. The Open Nutraceuticals Journal. 2014;7(1).

26. Eyileten C, Kaplon-Cieslicka A, Mirowska-Guzel D, Malek L, Postula M. Antidiabetic effect of brainderived neurotrophic factor and its association with inflammation in type 2 diabetes mellitus. Journal of diabetes research. 2017;2017.

27. Han J. Rare syndromes and common variants of the brain-derived neurotrophic factor gene in human obesity. Progress in molecular biology and translational science. 140: Elsevier; 2016. p. 7595.

28. Xu B, Xie X. Neurotrophic factor control of satiety and body weight. Nature Reviews Neuroscience. 2016;17(5):282-92.

29. Dooley LN, Ganz PA, Cole SW, Crespi CM, Bower JE. Val66Met BDNF polymorphism as a vulnerability factor for inflammation-associated depressive symptoms in women with breast cancer. Journal of affective disorders. 2016;197:43-50.

30. Wang Y, Zhang H, Li Y, Wang Z, Fan Q, Yu S, et al. BDNF Val66Met polymorphism and plasma levels in Chinese Han population with obsessive-compulsive disorder and generalized anxiety disorder. Journal of affective disorders. 2015;186:7-12.

31. He M, Wang J. Decreased serum brain-derived neurotrophic factor in Chinese patients with type 2 diabetes mellitus. Acta Biochim Biophys Sin. 2014;46(5):426-7.

32. Asghari G, Mirmiran P, Yuzbashian E, Azizi F. A systematic review of diet quality indices in relation to obesity. British Journal of Nutrition. 2017;117(8):1055-65.

33. Fung TT, McCullough ML, Newby P, Manson JE, Meigs JB, Rifai N, et al. Diet-quality scores and plasma concentrations of markers of inflammation and endothelial dysfunction-. The American journal of clinical nutrition. 2005;82(1):163-73.

34. Jiang R, Babyak MA, Brummett BH, Hauser ER, Shah SH, Becker RC, et al. Brain-derived neurotrophic factor rs6265 (Val66Met) polymorphism is associated with disease severity and incidence of cardiovascular events in a patient cohort. American heart journal. 2017;190:40-5.

35. Khakpouri S, Safari M, Ghazizadeh H, Parizadeh SMR, Nematy M, Tayefi M, et al. The relationship between the healthy eating index and an alternate healthy eating index with the risk factors for cardiovascular disease in a population from northeastern Iran. Translational Metabolic Syndrome Research. 2019;2(1):1-6.

36. Li J, Demirel A, Azuero A, McLain A, Yarar-Fisher C. The Relationship Between Healthy Eating Index2015 and Cardiometabolic Risk Factors in People with Long-Standing Spinal Cord Injury. Current Developments in Nutrition. 2020;4(Supplement_2):540-. 
37. Haghighatdoost F, Sarrafzadegan N, Mohammadifard N, Sajjadi F, Maghroon M, Boshtam M, et al. Healthy eating index and cardiovascular risk factors among Iranians. Journal of the American College of Nutrition. 2013;32(2):111-21.

38. Edalati S, Alipour B, Rashidkhani B. Association between Dietary Intake of Phytochemicals and hsCRP in Healthy Women from Tehran: a Holistic Approach Using Dietary Phytochemical Index. Nutrition and Food Sciences Research. 2018;5(3):11-6.

39. Vincent HK, Bourguignon CM, Taylor AG. Relationship of the dietary phytochemical index to weight gain, oxidative stress and inflammation in overweight young adults. Journal of human nutrition and dietetics. 2010;23(1):20-9.

40. Zanella PB, August PM, Alves FD, Matté C, de Souza CG. Association of Healthy Eating Index and oxidative stress in adolescent volleyball athletes and non-athletes. Nutrition. 2019;60:230-4.

41. Halliwell $B$, Whiteman M. Measuring reactive species and oxidative damage in vivo and in cell culture: how should you do it and what do the results mean? British journal of pharmacology. 2004;142(2):231-55.

42. Maxwell S, Cruickshank A, Thorpe G. Red wine and antioxidant activity in serum. The Lancet. 1994;344(8916):193-4.

43. Pitsavos C, Panagiotakos DB, Tzima N, Chrysohoou C, Economou M, Zampelas A, et al. Adherence to the Mediterranean diet is associated with total antioxidant capacity in healthy adults: the ATTICA study-. The American journal of clinical nutrition. 2005;82(3):694-9.

44. Hassimotto NMA, Pinto MrDS, Lajolo FM. Antioxidant status in humans after consumption of blackberry (Rubus fruticosus L.) juices with and without defatted milk. Journal of agricultural and food chemistry. 2008;56(24):11727-33.

45. Modun D, Music I, Vukovic J, Brizic I, Katalinic V, Obad A, et al. The increase in human plasma antioxidant capacity after red wine consumption is due to both plasma urate and wine polyphenols. Atherosclerosis. 2008;197(1):250-6.

46. Kim J, Yang Y, Yang Y, Oh S, Hong Y-C, Lee E, et al. Diet quality scores and oxidative stress in Korean adults. European journal of clinical nutrition. 2011;65(12):1271-8.

47. Tiwari BK, Pandey KB, Abidi A, Rizvi SI. Markers of oxidative stress during diabetes mellitus. Journal of biomarkers. 2013;2013.

\section{Tables}

Due to technical limitations, table 1, 2, 3 is only available as a download in the Supplemental Files section.

\section{Figures}


A

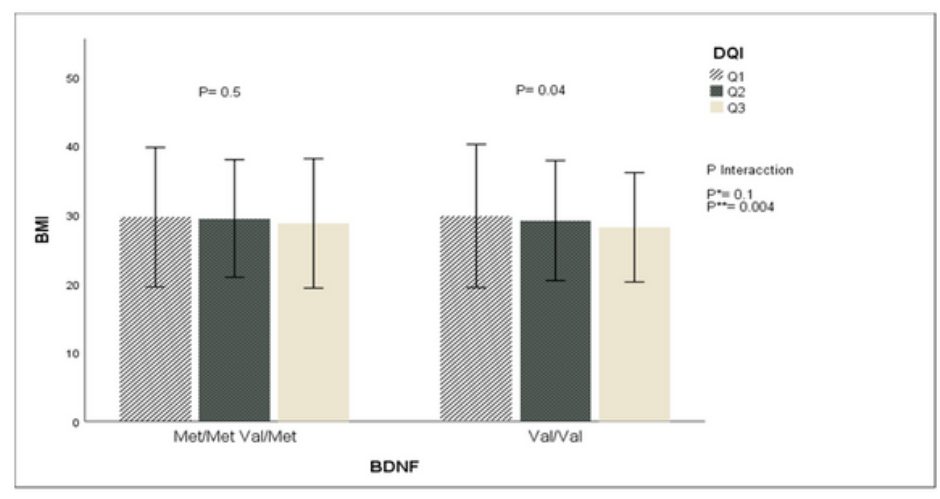

C

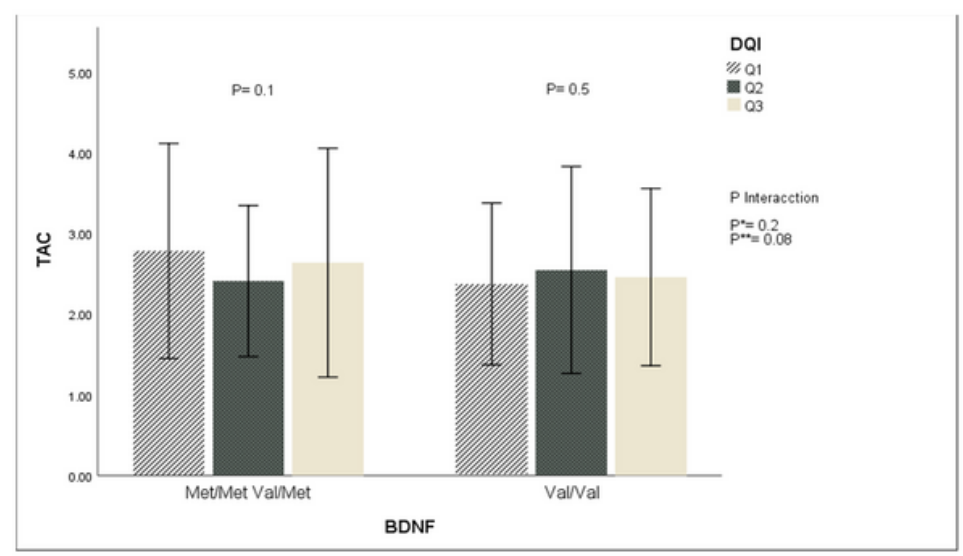

B

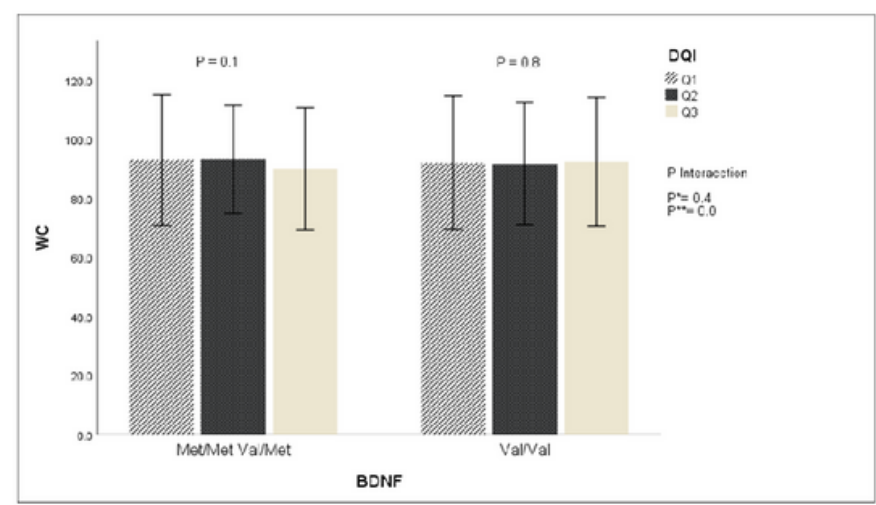

D

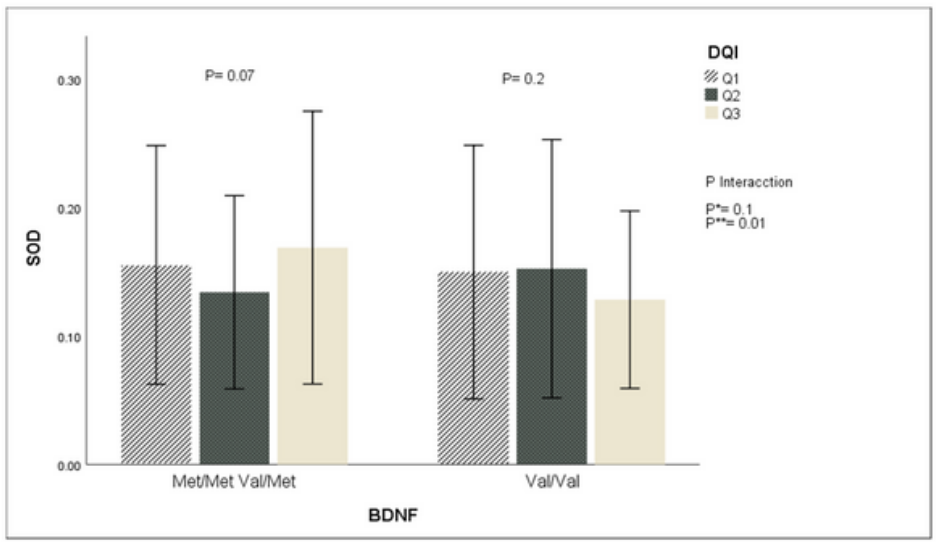

Figure 1

The interaction between BDNF Val66Met genotypes and quartiles of diet quality index (DQI) on: A: Body mass index (BMI), B: Waist circumference (WC), C: total antioxidant capacity (TAC), D: superoxide dismutase (SOD). Mean and Standard Error. $P$ values for the interaction obtained in two models using ANCOVA. $P^{*}$ : unadjusted; $P^{\star *}$ : adjusted for age, sexuality, smoking, alcohol consumption and physical activity. 


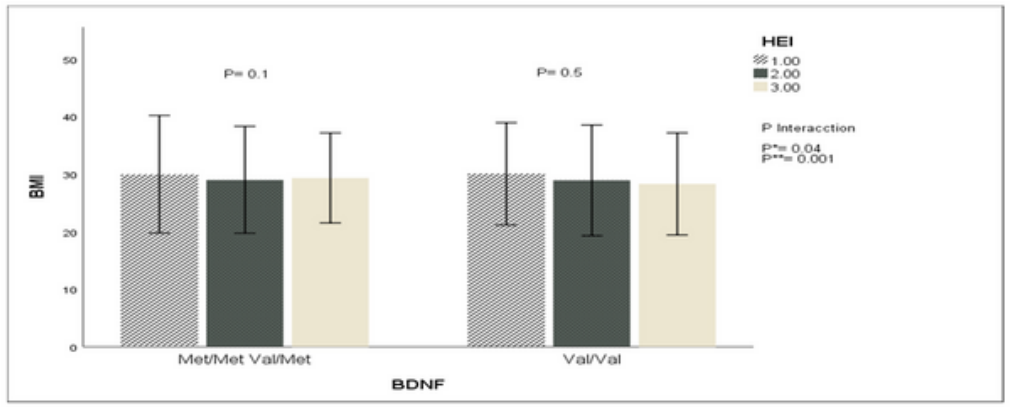

B

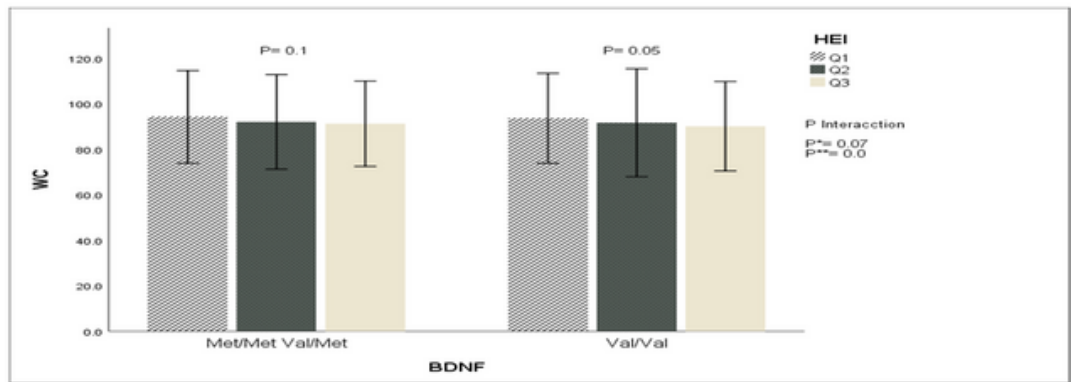

C

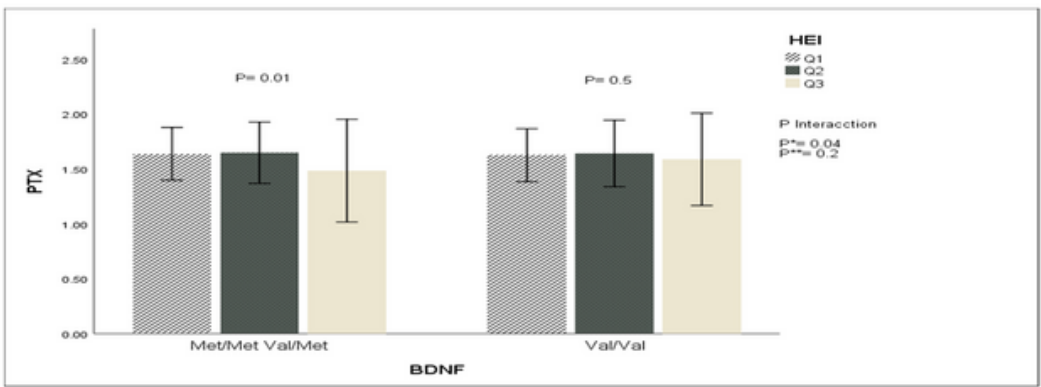

D

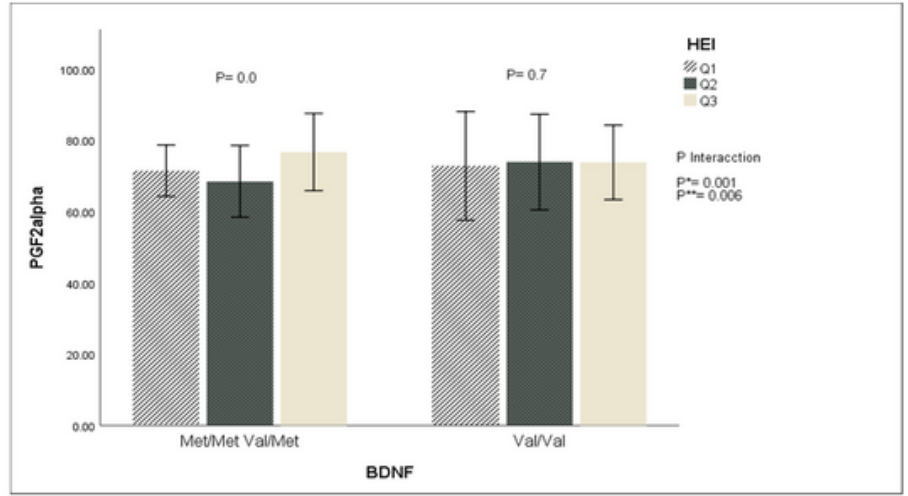

Figure 2

The interaction between BDNF Val66Met genotypes and quartiles of Healthy eating diet (HEI) on: A: Body mass index (BMI), B: Waist circumference (WC), C: Pentraxins (PTX), D: Prostaglandin F2 Alpha (PGF2). Mean and Standard Error. $P$ values for the interaction obtained in two models using ANCOVA. $P *$ : unadjusted; $P^{\star \star}$ : adjusted for age, sexuality, smoking, alcohol consumption and physical activity. 


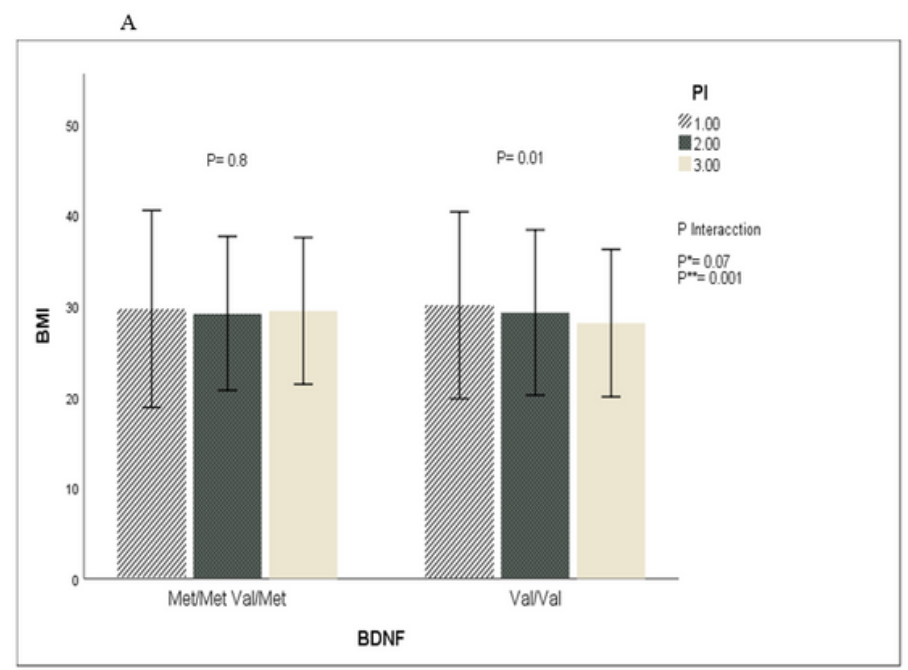

B

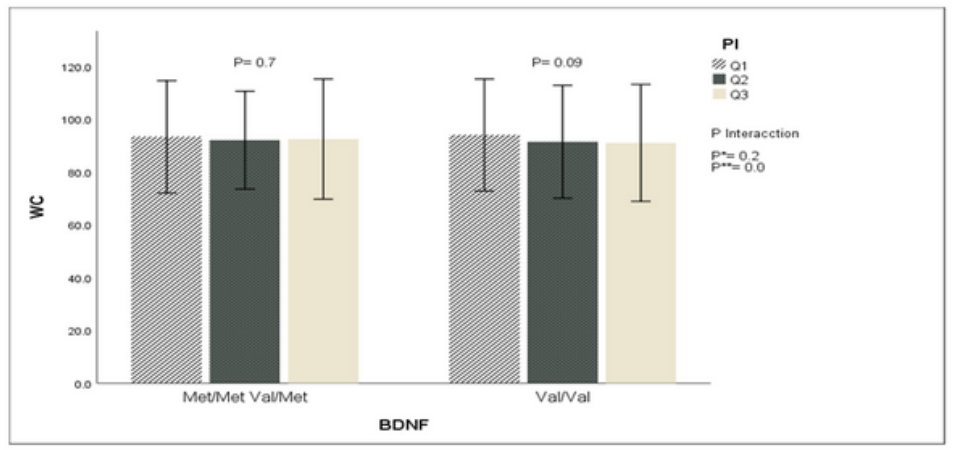

Figure 3

The interaction between B DNF Val66Met genotypes and quartiles of Dietary phytochemical index (PI) on: A: Body mass index (BMI), B: Waist circumference (WC). Mean and Standard Error. P values for the interaction obtained in two models using ANCOVA. $P^{\star}$ : unadjusted; $P^{\star \star}$ : adjusted for age, sexuality, smoking, alcohol consumption and physical activity.

\section{Supplementary Files}

This is a list of supplementary files associated with this preprint. Click to download.

- Table.docx

- STROBEnutchecklist.docx 